\title{
DOUBLY SLICED KNOTS WHICH ARE NOT THE DOUBLE OF A DISK
}

\author{
L. SMOLINSKY
}

\begin{abstract}
In this paper we show that double disk knots can be distinguished from general doubly sliced knots in dimensions $4 n+1$.
\end{abstract}

A double disk knot is formed by unioning two identical disk knots along their boundary. J. P. Levine has demonstrated that these knots are all doubly sliced [9], i.e., they can be realized as a slice of the trivial knot. Spun knots, high dimensional ribbon knots [2], and Sumners' knots constructed in [13] are all examples of double disk knots.

In [9] Levine also gives an example of a classical knot and a 2-knot that are doubly sliced but not the double of a disk. In this paper we show that doubly sliced knots are distinct from double disk knots in dimensions $4 n+1$. Our method of distinguishing double disk knots produces obstructions from the Casson-Gordon invariants. This paper is the main result of the author's Ph.D. thesis and he wishes to thank his advisor, J. P. Levine, for his help and encouragement.

An $n$ dimensional knot is a codimension two spherical knot or a smooth oriented pair $\left(S^{n+2}, K\right)$ where $K$ is a submanifold which is homeomorphic to $S^{n}$. An $n$ dimensional disk knot is a smooth oriented pair $\left(B^{n+2}, D\right)$ where $D$ is homeomorphic to the $n$-disk and $\partial B^{n+2} \cap D=\partial D$.

We apply disk knots to the study of knots in two distinct ways. First, the boundary of an $n$-disk knot $\left(B^{n+2}, D\right)$ is the $(n-1)$-knot $\left(\partial B^{n+2}, \partial D\right)$. Second, we may join two $n$-disk knots that have diffeomorphic boundaries along their boundaries. Here we obtain an $n$-knot. If two disk knots $\left(B_{1}^{n+2}, D_{1}\right)$ and $\left(B_{2}^{n+2}, D_{2}\right)$ have diffeomorphic boundaries by some orientation preserving diffeomorphism, $f: \partial\left(B_{1}, D_{1}\right)$ $\rightarrow \partial\left(B_{2}, D_{2}\right)$, then we may form the $n$-knot

$$
-\left(B_{1}, D_{1}\right) \cup_{f}\left(B_{2}, D_{2}\right)=\left(-B_{1} \cup_{f} B_{2},-D_{1} \cup_{f} D_{2}\right) .
$$

Given an $n$-disk knot $\left(B^{n+2}, D\right)$, one can construct an $(n+1)$-disk knot $\Sigma(B, D)$ called the suspension of $D$. In the P.L. category the suspension of $D$ may be realized as $\left(B^{n+2} \times I, D \times I\right)[9]$. We use the smooth version, obtained by rounding the corners. The boundary of $\Sigma D$ is $\left(-B^{n+2},-D\right) \cup_{I}\left(B^{n+2}, D\right)$, the $n$-knot formed by doubling the disk $\left(B^{n+2}, D\right)$. Knots formed by doubling a disk are called double disk knots.

A knot which is the boundary of a disk knot is called null cobordant. Some knots bound particularly nice disk knots and are slices of the trivial knot. The disk knot $\left(B^{n+2}, D\right)=D^{n+2, n}$ is invertible if there exists a disk $\operatorname{knot}\left(B^{n+2}, \Delta\right)=\Delta^{n+2, n}$ and a diffeomorphism, $f: \partial D^{n+2, n} \rightarrow \partial \Delta^{n+2, n}$ such that $-D^{n+2, n} \cup_{f} \Delta^{n+2, n}$ is the

Received by the editors September 23, 1985 and, in revised form, December 20, 1985.

1980 Mathematics Subject Classification (1985 Revision). Primary 57Q45, 57Q60. 
trivial knot. Knots which bound invertible disks are called doubly-null-cobordant or doubly sliced [13]. "Doubly" refers only to a higher order of slicing and is not an operational word as in double disk knot.

Our observations rely upon the Casson-Gordon invariants [4] as generalized by Gilmer [5]. We now define these invariants.

Let $M$ be a $(2 k-1)$-manifold and $d$ an integer. Isomorphism classes of $Z_{d^{-}}$ covers of $M$ with a specified generator for the covering translations correspond to elements of $\left[M, B Z_{d}\right]=H^{1}\left(M, Z_{d}\right)=\operatorname{Hom}\left(H_{1}(M), Z_{d}\right)$. If $\psi \in \operatorname{Hom}\left(H_{1}(M), Z_{d}\right)$ we get $\langle\tilde{M}, \tau\rangle$, such a covering with a generator. There exists an $n$ and a $2 k$-manifold with boundary $W$ such that $\langle\tilde{W}, T\rangle$ is a $Z_{d}$ cover of $W$ and $\partial\langle\tilde{W}, T\rangle=n \cdot\langle\tilde{M}, \tau\rangle$,

$$
\partial\left(\begin{array}{c}
\tilde{W} \\
\downarrow \\
W
\end{array}\right)=n\left(\begin{array}{c}
\tilde{M} \\
\downarrow \\
M
\end{array}\right) .
$$

This fact is essentially that $\Omega_{2 k-1}\left(B Z_{d}\right)$ is torsion. Let $\bar{H}_{k}(W, \psi)=e^{2 \pi i / d}$ eigenspace of $T_{*}$ in $H_{k}(\tilde{W}) \otimes \mathbf{C}$. If $k$ is even then the intersection form, $\langle x, y\rangle=x \cdot y$, is Hermitian and if $k$ is odd the form $\langle x, y\rangle=i x \cdot y$ is Hermitian. Let $\bar{\sigma}(W, \psi)$ denote the signature of $\left.\langle\rangle\right|_{,\bar{H}_{k}(W, \psi)}$. We define the Casson-Gordon invariant as

$$
\sigma(M, \psi)=\frac{1}{n}(\bar{\sigma}(W, \psi)-\sigma(W))
$$

Gilmer [5] shows this invariant to be well defined.

An $m$ component link of dimension $n$ or an $m$-link is an ordered collection of $m$ disjoint smooth oriented submanifolds of $S^{n+2}$, each of which is homeomorphic to $S^{n}$. We assume $n>2$ and our links will be ordered. A link is denoted by $L$ or $\left(S^{n+2} ; L_{1}, \ldots, L_{m}\right)$. Every $m$-link is bounded by a Seifert surface, $W$. A link is a boundary link if it has an $m$-component Seifert surface $W=W_{1} \cup \cdots \cup W_{m}$ with $\partial W_{i}=L_{i}$.

The following construction and invariant appears in $[\mathbf{1 1}]$ and is further developed in $[\mathbf{1 2}]$. We state the needed results. These results generalize [15].

Let $V_{1}, \ldots, V_{m} \subset D^{n+3}$ be disjoint codimension two submanifolds with trivial normal bundles such that $V_{i} \cap \partial D^{n+3}=\partial V_{i}=L_{i}$. Denote such an ordered set by $\left(D^{n+3} ; V_{1}, \ldots, V_{m}\right)$ and call this collection a special $m$-tuple. For every link there are special $m$-tuples. By Alexander duality $H_{1}\left(D^{n+3}-\left(V_{1} \cup \cdots \cup V_{m}\right)\right)=Z^{m}$, so let $\mu_{i}$ be the meridian to $V_{i}$. Let $G=Z_{a_{1}} \oplus \cdots \oplus Z_{a_{m}}$. We really wish to consider $m$-tuples of cyclic groups $\left.Z_{a_{1}}, \ldots, Z_{a_{m}}\right)$. The order is important and we indicate it by ordering the summands of $G$. We also choose preferred generators for $G$; let $g_{i}$ be the generator of $Z_{a_{i}}$.

THEOREM 1. Let $\left(D^{n+3} ; V_{1}, \ldots, V_{m}\right)$ be a special $m$-tuple and let $G=Z_{a_{1}} \oplus$ $\cdots \oplus Z_{a_{m}}$, one summand corresponding to one submanifold of $D^{n+3}$. Then there is a canonical $G$-manifold $M_{V}$ that has the following properties:

(1) $M \stackrel{\pi}{\rightarrow} M / G=D^{n+3}$.

(2) $M-\pi^{-1}\left(V_{1} \cup \cdots \cup V_{m}\right) \rightarrow D^{n+3}-\left(V_{1} \cup \cdots \cup V_{m}\right)$ is the regular $G$-covering space arising from the map

$$
\varphi: H_{1}\left(D^{n+1}-\left(V_{1} \cup \cdots \cup V_{m}\right)\right) \rightarrow G, \quad \varphi\left(\mu_{i}\right)=g_{i} .
$$

(3) $\pi^{-1}\left(V_{i}\right) \stackrel{\pi}{\rightarrow} V_{i}$ is a regular covering space with group $G / Z_{a_{i}}$. 
Suppose $n=2 q-1$. Let $\langle$,$\rangle again denote the Hermitian pairing on H_{q+1}\left(M_{V}\right) \otimes$ C. If $\omega_{i}$ is an $a_{i}$ th root of unity we let $E=\bigcap_{i=1}^{m}\left(\omega_{i}\right.$ eigenspace of $\left.g_{i *}\right)$. Define the link invariant $\operatorname{sig}_{L}\left(\omega_{1}, \ldots, \omega_{m}\right)$ as signature $\left(\left.\langle\rangle\right|_{E},\right)$. If $\chi$ is the irreducible characer $\chi\left(g_{i}\right)=\omega_{i}$, the inner product on characters is [, ] and $\operatorname{Sign}\left(G, M_{V}\right)$ is the character from the $G$-signature representation $[\mathbf{3}]$, then

$$
\operatorname{sig}_{L}\left(\omega_{1}, \ldots, \omega_{m}\right)=\left[\operatorname{Sign}\left(G, M_{V}\right), \chi\right] .
$$

This invariant is independent of the chosen $V_{i}$ 's and is a generalization of the LevineTristram signature function, $\sigma_{L}()[\mathbf{8}, \mathbf{1 4}]$. In particular, $\sigma_{L}(\omega)=\operatorname{sig}_{L}(\omega, \ldots, \omega)$.

We suppose $L$ is a $(2 q-1)$-dimensional boundary link and $\hat{V}=\hat{V}_{1} \cup \cdots \cup \hat{V}_{m}$ is a collection of disjoint Seifert surfaces for the $L_{i}$. Let $S=\left(A_{i j}\right)$ be a Seifert matrix for $\hat{V}$. The matrix $S$ is composed of blocks of matrices. A diagonal block $A_{i i}$ is an $l_{i} \times l_{i}$ Seifert matrix for $\hat{V}_{i}$. The off-diagonal blocks record the linking information between the various $\hat{V}_{i}$. More details appear in [7].

THEOREM 2. If $\omega_{1}, \ldots, \omega_{m}$ is a collection of roots of unity and $L$ is the link described above, then

$$
\operatorname{sig}_{L}\left(\omega_{1}, \ldots, \omega_{m}\right)= \begin{cases}\operatorname{sign}\left(i(I-W)\left(-S W^{-1}-S^{T}\right)\right), & q \text { even } \\ \operatorname{sign}\left((I-W)\left(-S W^{-1}+S^{T}\right)\right), & q \text { odd }\end{cases}
$$

where

$$
W=\left(\begin{array}{cccccc}
{\left[\omega_{1}\right]} & & & & & \\
& & {\left[\omega_{2}\right]} & & 0 & \\
& 0 & & \ddots & & \\
& & & & & {\left[\omega_{m}\right]}
\end{array}\right), \quad\left[\omega_{i}\right]=\omega_{i} I_{l_{i} \times l_{i}} .
$$

We also remark that if $L$ is a boundary link then $\operatorname{sig}_{L}$ is an invariant of its boundary link cobordism class and if $L$ is null boundary link cobordant then $\operatorname{sig}_{L} \equiv$ 0 .

Detecting double disk knots. If $K$ is a knot and $D$ a disk knot we write $N_{K}$ and $N_{D}$ to denote the cyclic cover of the sphere or disk branched along the knot or disk knot which corresponds to the map $H_{1}$ (exterior) $\rightarrow Z_{d}$ by $\mu \rightarrow[1]$.

THEOREM 3. Let $\left(S^{2 q+1}, K\right)$ be the double of the disk knot $\left(B^{2 q+1}, \Delta\right)$. Also let $d$ and $a$ be positive integers. If $N_{K}$ is the $d$-fold branched cyclic cover of $\left(S^{2 q+1}, K\right)$ then there is a direct sum decomposition $H_{1}\left(N_{K}\right)=A \oplus B$ satisfying:

(1) There is an epimorphism $A \rightarrow B$.

(2) If $\phi: H_{1}\left(N_{K}\right) \rightarrow Z_{a}$ is a map such that $\left.\phi\right|_{B}=0$ then $\sigma\left(N_{K}, \phi\right)=0$.

REMARK. The obstructions in Theorem 3 differ from Ruberman's obstructions [10] on three points. First, the existence of an epimorphism allows us to distinguish summands of $H_{1}\left(N_{K}\right)$. Second, for the Casson-Gordon invariant bound, Ruberman shows $\left|\sigma\left(N_{K}, \phi\right)\right| \leq \operatorname{dim}\left(H_{q+1}\left(N_{K} ; Z_{a}\right)\right)$ while ours is zero. Third, Ruberman must assume $a=p^{r}$ for some prime $p$ while our $a$ is arbitrary.

ProOF. According to $[9]$ every suspension in invertible. Let $\left(B^{2 q+2}, D\right)$ be an inverse to $\Sigma \Delta$ so that $D \cup_{f} \Sigma \Delta$ is unknotted. The usual Mayer-Vietoris sequence argument $[6]$ yields

$$
0=H_{2}\left(S^{2 q+2}\right) \rightarrow H_{1}\left(N_{K}\right) \rightarrow H_{1}\left(N_{\Sigma \Delta}\right) \oplus H_{1}\left(N_{D}\right) \rightarrow H_{1}\left(S^{2 q+2}\right)=0
$$


since $N_{\text {unknot }}=S^{2 q+2}$. Let $B=\operatorname{Ker}\left\{H_{1}\left(N_{K}\right) \stackrel{i_{*}}{\rightarrow} H_{1}\left(N_{\Sigma \Delta}\right)\right\} \approx H_{1}\left(N_{D}\right)$ and $A=\operatorname{Ker}\left\{H_{1}\left(N_{K}\right) \rightarrow H_{1}\left(N_{D}\right)\right\} \approx H_{1}\left(N_{\Sigma \Delta}\right)$. So $H_{1}\left(N_{K}\right)=A \oplus B$.

Property 1. Consider the sequence of the pair $N_{\partial \Delta} \subset N_{\Delta}$,

(i) $H_{1}\left(N_{\partial \Delta}\right) \rightarrow H_{1}\left(N_{\Delta}\right) \stackrel{p_{*}}{\rightarrow} H_{1}\left(N_{\Delta}, N_{\partial \Delta}\right) \rightarrow 0$

where $N_{\partial \Delta}$ is the $d$-fold branched cover of $\left(\partial B^{2 q+1}, \partial \Delta\right)$. First note that $N_{\Sigma \Delta}$ is homeomorphic to $I \times N_{\Delta}$ and the composite map $N_{\Delta} \rightarrow N_{K} \rightarrow N_{\Sigma \Delta}$ is, up to a homeomorphism of $N_{\Sigma \Delta}, N_{\Delta} \rightarrow 1 \times N_{\Delta} \subset I \times N_{\Delta}$.

Consider the diagram,

$$
\begin{aligned}
& 0 \\
& \uparrow \\
& H_{1}\left(N_{K}, N_{\Delta}\right) \\
& 0 \rightarrow B \rightarrow H_{1}\left(N_{K}\right) \rightarrow H_{1}\left(N_{\Sigma \Delta}\right) \rightarrow 0 \\
& \begin{array}{c}
\uparrow \\
H_{1}\left(N_{\Delta}\right) \\
\uparrow \\
0
\end{array}
\end{aligned}
$$

A simple diagram chase yields that $f: B \rightarrow H_{1}\left(N_{K}, N_{\Delta}\right)$ is an isomorphism. By excision, $H_{1}\left(N_{K}, N_{\Delta}\right) \approx H_{1}\left(N_{\Delta}, N_{\partial \Delta}\right)$ and so by $p_{*}$ in (i) we have

$$
A \approx H_{1}(N) \stackrel{p_{*}}{\rightarrow} H_{1}\left(N_{\Delta}, N_{\partial \Delta}\right) \approx B
$$

is an epimorphism.

Property 2 . The sequence $0 \rightarrow B \rightarrow H_{1}\left(N_{K}\right) \stackrel{i_{*}}{\rightarrow} H_{1}\left(N_{\Sigma \Delta}\right) \rightarrow 0$ is split exact. So if $\phi: H_{1}\left(N_{K}\right) \rightarrow Z_{a}$ is zero on $B$ then $\phi$ extends to $\psi: H_{1}\left(N_{\Sigma \Delta}\right) \rightarrow Z_{a}$. The maps $\phi$ and $\psi$ represent classes in $H^{1}\left(N_{K} ; Z_{a}\right)$ and $H^{1}\left(N_{\Sigma \Delta} ; Z_{a}\right)$ and are related by $i^{*} \psi=\phi$. Since $H^{1}\left(X ; Z_{a}\right)=\left[X ; B Z_{a}\right]$, we get covering spaces $\tilde{N}_{K}$ and $\tilde{N}_{\Sigma \Delta}$ so that

$$
\begin{gathered}
\tilde{N}_{K} \\
\downarrow \\
N_{K}
\end{gathered}=\partial\left(\begin{array}{c}
\tilde{N}_{\Sigma \Delta} \\
\downarrow \\
N_{\Sigma \Delta}
\end{array}\right) .
$$

$N_{\Sigma \Delta}$ is homeomorphic to $N_{\Delta} \times I$ so the covering

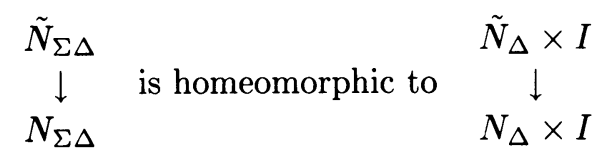

for some covering $\tilde{N}_{\Delta} \rightarrow N_{\Delta}$. Now, $\sigma\left(N_{K}, \phi\right)=\bar{\sigma}\left(N_{\Sigma \Delta}, \phi\right)-\sigma\left(N_{\Sigma \Delta}\right)$ which is zero, since both $\tilde{N}_{\Sigma \Delta}$ and $N_{\Sigma \Delta}$ are homeomorphic to products and all intersections are zero.

Doubly sliced knots. We now give a construction which produces examples of doubly sliced knots (compare with $[\mathbf{1 0}])$. Let $\left(S^{n+2} ; L_{1}, L_{2}\right)=L$ be a link with $\pi_{1}$ (exterior) $=Z * Z$. Imbed $L \times[-1,1]$ in $S^{n+2}$ using the unique (up to homotopy) untwisted normal vector field to $L$. Let $r$ and $s$ be integers and $p_{1}, p_{2} \in L_{1}$ and $q_{1}, q_{2} \in L_{2}$. We form a class of $\operatorname{knots} K(L ; r, s)$. Connect $p_{1} \times 1$ to $p_{1} \times(-1)$ 


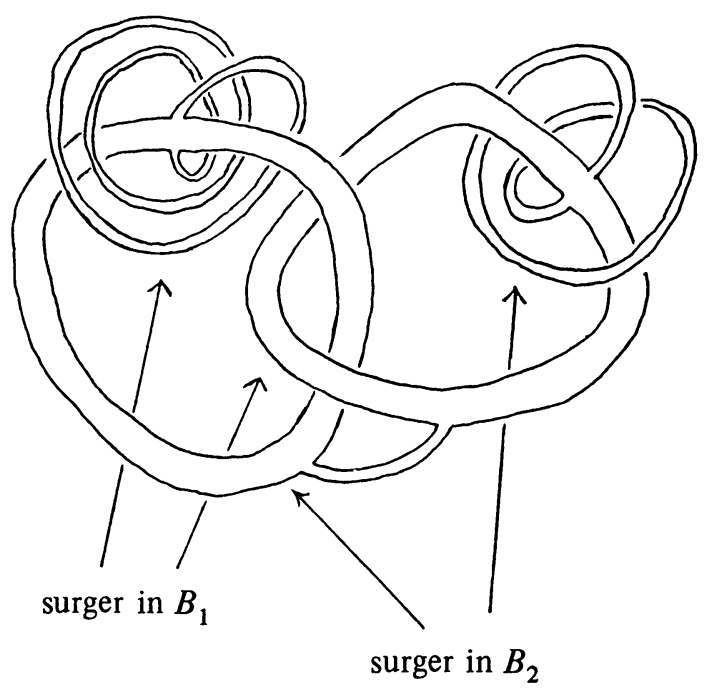

FIGURE 1

by a one handle which follows the meridian to $L_{1}$ for $r+\frac{1}{2}$ times around $L_{1} \times 0$ and connect $q_{1} \times 1$ to $q_{1} \times(-1)$ by a 1-handle which wraps around $L_{2} \times 0 s+\frac{1}{2}$ times following the meridian. The 1-handles are imbedded without twisting, i.e., the induced framing on $p_{1} \times I$ or $q_{1} \times I$ union the core of the handle is trivial and extends over a disk. Denote these 1-handles $h_{1}^{1}$ and $h_{2}^{1}$. Add a third 1-handle which connects $p_{2} \times 1$ to $q_{2} \times 1$. This construction depends upon the path from $p_{2} \times 1$ to $q_{2} \times 1$ and for each path we get a knot

$$
K=\partial\left(L_{1} \times I \cup L_{2} \times I \cup\right. \text { the three 1-handles). }
$$

The bounded manifold (i.e., the handlebody) is a Seifert surface for $K$ and is diffeomorphic to $\left(S^{n} \times S^{1}-D^{n+1}\right) \#_{\partial}\left(S^{n} \times S^{1}-D^{n+1}\right)$.

Proposition 4. (1) $K$ is a slice knot.

(2) If the knots $\left(S^{n+2}, L_{1}\right)$ and $\left(S^{n+2}, L_{2}\right)$ are slice knots then $K$ is a doubly sliced knot.

Proof. The two 1-handles, $h_{1}^{1}$ and $h_{2}^{1}$, may be surgered in $B^{n+3}$. The surgery changes each $S^{n} \times S^{1}-D^{n}$ into $D^{n+2}$ and we get $D^{n+2} \#_{\partial} D^{n+2}$ as a spanning disk for $K$.

(2) If $L_{1}$ and $L_{2}$ are slice knots then we can surger $L_{1}$ in $B_{1}^{n+3}$ and $L_{2}$ in $B_{2}^{n+3}$. We can also surger $h_{2}^{1}$ in $B_{1}^{n+3}$ and $h_{1}^{1}$ in $B_{2}^{n+3}$ (see Figure 1). The result is a knot in $S^{n+3}=B_{1} \cup B_{2}$ of which $\left(\partial B_{1}, K\right)$ is a slice. The trace of the above surgeries is an $(n+3)$-disk and so the knot in $S^{n+3}$ is trivial. Therefore $K$ is doubly sliced.

We wish to apply Theorem 3 to the knots $K(L ; r, s)$ so we now confront the problem of computing the associated Gasson-Gordon invariants.

Construction of the 2-fold cover. We denote by $N$ the 2 -fold branched cyclic cover of $K$. Let $\hat{V}$ denote the Seifert surface $V$ with its interior pushed into $\stackrel{\circ}{B}^{2 q+2}$. If $W$ is the 2-fold branched cyclic cover of $\left(B^{2 q+2}, \hat{V}\right)$ then $\partial W=N$. 


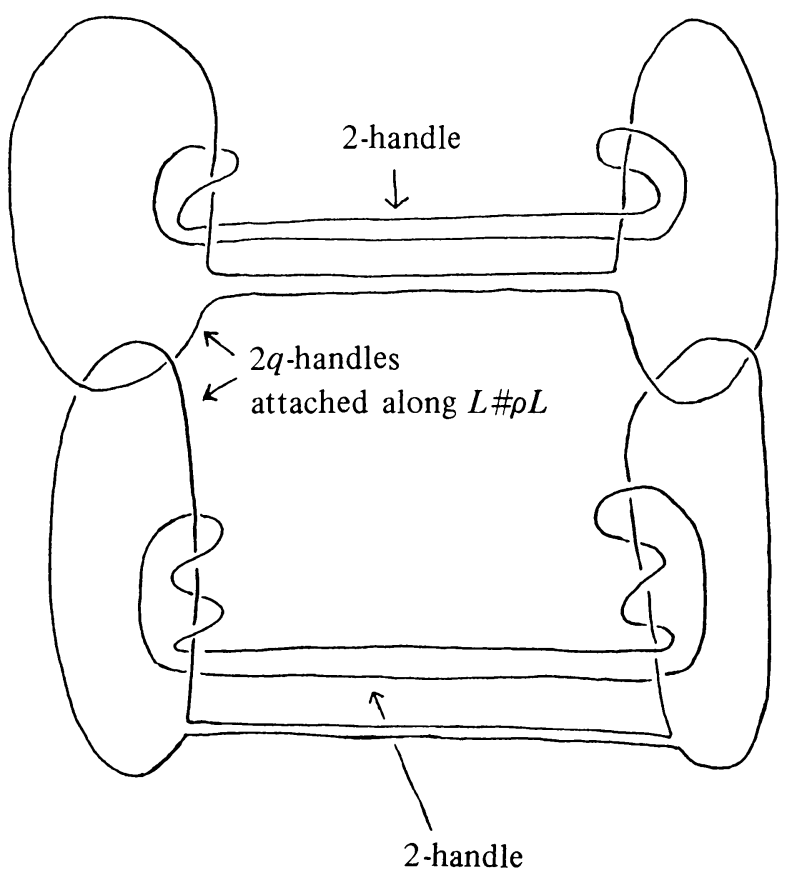

Surgery on a Link Description

\section{FIGURE 2}

The following description of $W$ is from $[\mathbf{1}]$ where a detailed analysis is given. $W$ is constructed from two copies of the $(2 q+2)$-ball, $B$ and $B^{\prime}$, both containing copies of the Seifert surface, $V$ and $V^{\prime}$, in their boundaries. Identify the Seifert surfaces to obtain $W$. These identifications are accomplished by identifying one handle at a time. As a handlebody, $V$ is constructed with two 0 -handles, $p$ and $q$; three 1-handles, an $S^{1}$ at $p$, an $S^{1}$ at $q$ and an arc from $p$ to $q$; and two $(2 q-1)$-handles, $L_{1}$ and $L_{2}$. Identify $p$ and $p^{\prime}$ with a 1 -handle and $q$ with $q^{\prime}$. Next identify the 1-handle between $p$ and $q$ with the corresponding 1-handle in $\partial B^{\prime}$. This partially constructed $W$ is a $2 q+2$ ball. To obtain $W$ we add 2 -handles and $2 q$-handles. The $2 q$-handles are attached along $L \# \rho L$ where $\rho\left(S^{2 q+1}, L\right)=\left(S^{2 q+1},-L\right)$. One 1-handle attaches along an $S^{1}$ which links $2 r+1$ times with $L_{1} \# \rho L_{1}$ and does not link with $L_{2} \# \rho L_{2}$. The other 1-handle links $2 s+1$ times with $L_{2} \# \rho L_{2}$ and does not link with $L_{1} \# \rho L_{1}$. If $2 r+1=a$ and $2 s+1=b$ then $H_{1}(N)=Z_{a} \oplus Z_{b}$. We have a "surgery on a link" description of $N$ (see Figure 2).

Computation of Casson-Gordon invariants. We use the $G$-signature theorem to compute Casson-Gordon invariants. The use of the $G$-signature theorem for this type of invariant is documented in $[\mathbf{3}, \S 7]$ and specifically for the Casson-Gordon invaraint in $[\mathbf{4}$ and $\mathbf{5}]$.

If $C$ is a closed $2 q+2$ dimensional $Z_{d}$-manifold so that $C / Z_{d}$ is a manifold then the $G$-signature theorem dictates a formula $F\left(C, Z_{d}\right)$ such that

$$
F\left(C, Z_{d}\right)+\sigma\left(C / Z_{d}\right)=\left.\operatorname{sign}\langle,\rangle\right|_{E_{1}(C)}
$$


where $E_{r}(C)=e^{2 \pi r i / d}$ eigenspace of the generator in $H_{q+1}(C) \otimes \mathbf{C}$ and $F\left(C, Z_{d}\right)$ is a formula involving only data about the fixed points of elements other than the identity and their normal bundles. This formula $F\left(C, Z_{d}\right)$ may be defined abstractly on any $Z_{d}$-manifold whether or not it is closed.

If $\phi: H_{1}\left(N^{2 q+1}\right) \rightarrow Z_{d}$ is realized by the covering space $\tilde{N} \rightarrow N$ then $\sigma(N, \phi)$ may be computed using a bounding $Z_{d}$-manifold $\hat{M}$ where $\hat{M} \rightarrow \hat{M} / Z_{d}=M$ and $\partial \hat{M} \rightarrow \partial M$ is the covering $\hat{N} \rightarrow N$. An argument similar to that in [4] yields,

$$
\sigma(N, \phi)=\left.\operatorname{sign}\langle,\rangle\right|_{E_{1}(\hat{M})}-\sigma(M)-F\left(\hat{M}, Z_{d}\right) .
$$

We compute $F\left(\hat{M}, Z_{d}\right)$ for certain types of branched covers but not the standard branched cyclic covers. Let $G=Z_{d_{1}} \oplus \cdots \oplus Z_{d_{m}}$ with $\left(d_{i}, d_{j}\right)=1$ if $i \neq j$. Let $|G|=d$ so that $G=Z_{d}$. Further, write $g_{i}$ for the generator of $Z_{d_{i}}$ and $\tau$ for $\prod_{i=1}^{m} g_{i}$. We wish our branched $G$-covers, $p: \tilde{M} \rightarrow M$, to satisfy the following properties:

(1) There exist $V_{i} \subset M, i \leq m$, disjoint closed codimension two submanifolds.

(2) The fixed points of $g_{i}$ are equivariantly $p^{-1}\left(V_{i}\right)=V_{i} \times G / Z_{d_{i}}$.

(3) $\nu\left(p^{-1}\left(V_{i}\right)\right)$ is a plane bundle and the action of $g_{i}$ is multiplication by a primitive $d_{i}$ th root of unity.

For integers $s$ and $r$ we let $s \equiv s_{i} \bmod d_{i}, s_{i}<d_{i} ; r \equiv r_{i} \bmod d_{i}, r_{i}<d_{i}$ and $\overline{s_{i} r_{i}} \equiv s_{i} r_{i} \bmod d_{i}, \overline{s_{i} r_{i}}<d_{i}$. The primitive $d$ th root of unity may be written uniquely as $\omega=e^{2 \pi i / d}=\omega_{1} \omega_{2} \cdots \omega_{m}$ where $\omega_{i}=e^{\left(2 \pi i / d_{i}\right) p_{i}}$ is a $d_{i}$ th root of unity.

LEMMA 5. Let $\tilde{M}^{2 q+2} \rightarrow M^{2 q+2}$ be a closed branched $Z_{d}$-cover as described above, so $\tau$ generates the covering translations. Then,

$$
\left.\operatorname{sign}\langle,\rangle\right|_{E_{r}(\tilde{M})}=\sigma(M)-\sum_{i} 2^{2 q} \frac{d_{i}-2 \overline{p_{i} r_{i}}}{d_{i}} \sigma\left(V_{i}\right) .
$$

PROOF. Let $\chi$ be the character so that $\chi(\tau)=\omega^{r}$. Then,

$$
\begin{aligned}
\left.\operatorname{sign}\langle,\rangle\right|_{E_{r}(\tilde{M})} & =[\operatorname{Sign}(G, \tilde{M}), \chi]=\frac{1}{d} \sum_{s=0}^{d-1} \operatorname{Sign}\left(\tau^{s}, \tilde{M}\right) \overline{\chi\left(\tau^{s}\right)} \\
& =\frac{1}{d} \sum_{s=1}^{d-1} \operatorname{Sign}\left(\tau^{s}, \tilde{M}\right) \omega^{-r s}+\sigma(M)
\end{aligned}
$$

since $\operatorname{Sign}(I, \tilde{M})=\sigma(\tilde{M})$ and a transfer argument yields $\sigma(\tilde{M})=d \sigma(M)$. The calculation of the last sum is similar to the calculation in Lemma 2.1 of [4] or 3.4 in $[\mathbf{1 0}]$. We refer the reader to these references for details.

Proposition 6. Let $L \subset S^{2 q+1}$ be a boundary m-link and let $W=B^{2 q+2} \cup$ $\left\{h_{i}^{2}\right\} \cup\left\{h_{j}^{2 q}\right\}$ where the $h_{i}^{2}$ are 2-handles and the $h_{j}^{2 q}$ are $2 q$-handles attached along $L_{j}$. Let $M=\partial W$ and $\mu_{i} \in H_{1}(M)$ represent the ith meridian. If $\phi: H_{1}(M) \rightarrow G$ by $\phi\left(\mu_{i}\right)=g_{i}$ is a well defined map then for $0<r<d$ we have

$$
\sigma(M, r \phi)= \begin{cases}\operatorname{sig}_{L}\left(\omega_{1}^{r_{1}}, \ldots, \omega_{m}^{r_{m}}\right) & \text { if } q \text { is odd }, \\ \operatorname{sig}_{L}\left(\omega_{1}^{r_{1}}, \ldots, \omega_{m}^{r_{m}}\right)+\sum_{i=1}^{m} 2^{2 q} \frac{d_{i}-2 \overline{p_{i} r_{i}}}{d_{i}} \sigma\left(A_{i i}+A_{i i}^{T}\right) & \text { if } q \text { is even },\end{cases}
$$

where $A_{i i}$ is a Seifert matrix for $L_{i}$. 
The proof of this theorem proceeds in a similar fashion to Theorem 3.5 in $[\mathbf{1 0}]$ but uses our formula $F($,$) and the Z_{d_{1}} \oplus \cdots \oplus Z_{d_{m}}$ manifold associated to $L$ (see Theorem 1) instead of the branched $Z_{d}$ cover.

PROPOSITION 7. If $L \subset S^{2 q+1}$ is boundary sliced, then $\sigma(M, r \phi)=0$.

REMARK. More general results are possible than those of Propositions 6 and 7 but the proofs are more complicated and the results are not required in this paper. We still wish to note the following:

(1) If $L \subset S^{2 q+1}$ is any $m$-link and $A_{i i}$ is the matrix of a Seifert surface for the knot $\left(S^{2 q+1}, L_{i}\right)$ then the formulas of Proposition 6 are valid.

(2) If $L$ is composed of slice knots then $\sigma(M, r \phi)=\operatorname{sig}_{L}\left(\omega_{1}^{r_{1}} \cdots \omega_{m}^{r_{m}}\right)$.

We write $\Omega$ for an $m$-tuple of roots of unity, $\left(\omega_{1}, \ldots, \omega_{m}\right)$.

PROOF OF PROPOSITION 7. If $L$ is boundary sliced then $\operatorname{sig}_{L}(\Omega)=0$ for all $m$-tuples $\Omega$ (Theorem 2). Since each $\operatorname{knot}\left(S^{2 q+1}, L_{i}\right)$ is sliced all of its Seifert surfaces have signature zero.

\section{An example.}

PROPOSITION 8. Suppose $2 r+1=a$ and $2 s+1=b$ are distinct primes, $L \subset S^{2 q+1}$ is a 2-component boundary link with Seifert surface $V_{1} \cup V_{2}$ and $\sigma\left(V_{1}\right)=$ $\sigma\left(V_{2}\right)=0$. If $K \in K(L ; r, s)$ is a double disk knot then $\operatorname{sig}_{L \# \rho L}\left(\omega_{1}, \omega_{2}\right)=0$ for all $\omega_{1}$ an ath root of unity and $\omega_{2}$ a bth root of unity.

PROOF. Let $M$ be the 2-fold branched cyclic cover of $\left(S^{2 q+1}, K\right)$ as it was previously constructed. By Theorem $3, H_{1}(M)=A \oplus B$ and there is an epimorphism $A \rightarrow B$. But $H_{1}(M)=Z_{a} \oplus Z_{b}$ and $(a, b)=1$ so that the only possible decomposition is $A=Z_{a} \oplus Z_{b}$ and $B=0$. Therefore by (2) of Theorem 3, all the Casson-Gordon invariants of $M$ must vanish. Let $\phi: H_{1}(M) \rightarrow Z_{a} \oplus Z_{b}$ be $\phi\left(\mu_{1}\right)=(1,0)$ and $\phi\left(\mu_{2}\right)=(0,1)$. If $\omega_{1} \omega_{2}=e^{2 \pi r i / a b}$ then by Proposition 6 and the condition $\sigma\left(V_{i}\right)=\sigma\left(A_{i i}+A_{i i}^{T}\right)=0, \sigma(M, r)=\operatorname{sig}_{L \# \rho L}\left(\omega_{1}, \omega_{2}\right)$ so the theorem follows.

LEMMA 9. If the boundary $m$-link $J \subset S^{2 q+1}$ has Seifert matrix $\left(A_{i j}\right)$ then $\rho J$ has Seifert matrix $\left(B_{i j}\right)$ where $B_{i j}=(-1)^{q+1} A_{j i}^{T}$.

PROOF. $J$ and $\rho J$ have the same Seifert surface except for orientation. The + direction for $J$ is the - direction for $\rho J$. If $x$ and $y$ are $q$ th dimensional homology classes of the Seifert surface and $i_{+}$denotes the push off map in the + direction for $J$ then

$$
\lambda\left(x, i_{+} y\right)=\lambda\left(i_{-} x, y\right)=(-1)^{q+1} \lambda\left(y, i_{-} x\right)
$$

so $A_{i j}=(-1)^{q+1} B_{j i}^{T}$.

LEMMA 10. If $L$ is a boundary link in $S^{2 q+1}$ then $\operatorname{sig}_{\rho L}(\Omega)=(-1)^{q+1} \operatorname{sig}_{L}(\Omega)$.

PROOF. Let $W$ be a root of unity matrix as in Theorem 2 . We first show that $\operatorname{sig}_{\rho L}(\Omega)=\operatorname{sig}_{L}(\bar{\Omega})$. By Theorem 2 we can compute $\operatorname{sig}_{L}(\Omega)$ as $\sigma(i A)$ or $\sigma(A)$ where

$$
A=(I-W)\left(-S W^{-1}+(-1)^{q+1} S^{T}\right)
$$


and so by Theorem $2 \operatorname{sig}_{\rho L}(\Omega)=\sigma(B)$ or $\sigma(i B)$ where

$$
B=(I-W)\left(-(-1)^{q+1} S^{T} W^{-1}+S\right) .
$$

Now,

$$
\begin{aligned}
W^{-1} B W & =W^{-1}(I-W)\left(-(-1)^{q+1} S^{T} W^{-1}+S\right) W \\
& =\left(W^{-1}-I\right)\left(-(-1)^{q+1} S^{T}+S W\right)=\left(I-W^{-1}\right)\left(-S W+(-1)^{q+1} S^{T}\right)
\end{aligned}
$$

so $\operatorname{sig}_{L}(\bar{\Omega})=\operatorname{sig}_{\rho L}(\Omega)$. Now, if $X$ is Hermitian then $\sigma(X)=\sigma(\bar{X})$ since if $O X O^{*}=$ $R$ is a real matrix then $\bar{O} \bar{X} \bar{O}^{*}=\bar{R}=R$. If $q$ is odd

$$
\operatorname{sig}_{L}(\Omega)=\sigma(A)=\sigma(\bar{A})=\operatorname{sig}_{L}(\bar{\Omega})=\operatorname{sig}_{\rho L}(\Omega) .
$$

If $q$ is even

$$
\operatorname{sig}_{L}(\Omega)=\sigma(i A)=\sigma(\overline{i A})=-\sigma(i \bar{A})=-\operatorname{sig}_{L}(\bar{\Omega})=-\operatorname{sig}_{\rho L}(\Omega)
$$

Let $L$ be the simple boundary link given by the following matrix (see [7] to realize this matrix as a link):

$$
\left[\begin{array}{ll}
\left(\begin{array}{ll}
0 & 1 \\
0 & 0
\end{array}\right) & \left(\begin{array}{ll}
1 & 1 \\
1 & 1
\end{array}\right) \\
\left(\begin{array}{ll}
1 & 1 \\
1 & 1
\end{array}\right) & \left(\begin{array}{ll}
0 & 1 \\
0 & 0
\end{array}\right)
\end{array}\right] .
$$

Let $K$ be a knot of the form $K(L ; 2,1)$. Each component of $L$ is unknotted so by Proposition 4,K is doubly sliced. If $K$ is also a double disk knot then by Proposition $8, \operatorname{sig}_{L \# \rho L}(\omega, \eta)=0$ for $\omega$ a 5 th root of unity and $\eta$ a 3rd root of unity. Now, $\operatorname{sig}_{L}(\omega, \eta)$ is equal to

$$
\operatorname{sign}\left\{\left[\begin{array}{cccc}
0 & 1-\omega^{-1} & (1-\omega)\left(1-\eta^{-1}\right) & (1-\omega)\left(1-\eta^{-1}\right) \\
1-\omega & 0 & (1-\omega)\left(1-\eta^{-1}\right) & (1-\omega)\left(1-\eta^{-1}\right) \\
\left(1-\omega^{-1}\right)(1-\eta) & \left(1-\omega^{-1}\right)(1-\eta) & 0 & 1-\eta^{-1} \\
\left(1-\omega^{-1}\right)(1-\eta) & \left(1-\omega^{-1}\right)(1-\eta) & 1-\eta & 0
\end{array}\right]\right\}
$$

which is

$\operatorname{sign}\left\{\left[\begin{array}{cccc}-(1-\omega)\left(1-\omega^{-1}\right) & 0 & 0 & 0 \\ 0 & 1 & 0 & 0 \\ 0 & 0 & 1-(1-\omega)\left(1-\omega^{-1}\right)(1-\eta)\left(1-\eta^{-1}\right) & 0 \\ 0 & 0 & 0 & -(1-\eta)\left(1-\eta^{-1}\right)\end{array}\right]\right\}$

$\operatorname{sig}_{L}(\omega, \eta)$ is nonzero for $\eta=e^{2 \pi i / 3}$ and $\omega=e^{2 \pi \cdot 3 i / 5}$ since $\|1-\omega\|$ and $\|1-\eta\|$ are greater than 1. By Lemma $24, \operatorname{sig}_{L \# \rho L}(\omega, \eta)=2 \operatorname{sig}_{L}(\omega, \eta)$ and so $K$ is not a double disk knot.

\section{REFERENCES}

1. S. Akbulut and R. Kirby, Branched covers of surfaces in 4-manifolds, Math. Ann. 252 (1980), 111-132.

2. K. Asano, Y. Marumoto and T. Yanagawa, Ribbon knots and ribbon disks, Osaka J. Math. 18 (1981), 161-174. 
3. M. F. Atiyah and I. M. Singer, The index of elliptic operators: III, Ann. of Math. 87 (1968), 546-604.

4. A. Casson and C. Gordon, On slice knots in dimension three, Proc. Sympos. Pure Math. vol. 32, Amer. Math. Soc., Providence, R.I., 1978.

5. P. Gilmer, Configurations of surfaces in 4-manifolds, Trans. Amer. Math. Soc. 264 (1981), 353-380.

6. P. Gilmer and C. Livingston, On embedding 3-manifolds in 4-space, Topology 22 (1983), 241-252.

7. Ki Hyoung Ko, Seifert matrices and boundary link cobordisms, preprint.

8. J. Levine, Invariants of knot cobordism, Invent. Math. 8 (1969), 98-110.

9. __ Doubly sliced knots and doubled disk knots, Michigan Math. J. 30 (1983), 249-256.

10. D. Ruberman, Doubly sliced knots and the Casson-Gordon invariants, Trans. Amer. Math. Soc. 279 (1983), 569-588.

11. L. Smolinsky, Double disk knots and a link invariant, Ph.D. Thesis, Brandeis University, 1985.

12. $\ldots$ A generalization of the Levine-Tristram link invariant, in preparation.

13. D. W. Sumners, Invertible knot cobordisms, Comment. Math. Helv. 46 (1971), 240-256.

14. A. G. Tristram, Some cobordism invariants for links, Proc. Cambridge Philos. Soc. 66 (1969), 251-264.

15. O. Ja. Viro, Branched coverings of manifolds with boundary and link invariants. I, Math. USSR Izv. 7 (1973), 1239-1256.

16. D. Zagier, Equivariant Pontrjagin classes and applications to orbit spaces, Lecture Notes in Math., vol. 290, Springer-Verlag, New York, 1972.

Department of Mathematics, Yale University, Box 2155 Yale Station, New HAVEN, CONNECTICUT 06520 (Current address)

Department of Mathematics, Brandeis University, Waltham, MassachuSETTS 02254 